\title{
An Architecture For FACTS Controllers To Deal With Bandwidth Constrained Communication
}

\author{
Nilanjan Ray Chaudhuri, Member, IEEE, Debraj Chakraborty, Member, IEEE \\ and Balarko Chaudhuri, Member, IEEE
}

\begin{abstract}
Bandwidth constraints could have adverse impact on the flexible AC transmission system (FACTS) controllers relying on signals communicated from distant locations. An observer driven system copy (OSC) architecture is adopted here to deal with low data rates caused by the limited bandwidth availability. The basic idea is to use the knowledge of the nominal system to approximate its actual behavior during the intervals when data from the remote phasor measurement units (PMUs) is not available. This is corrected whenever the most recent states are obtained from the reduced order observer at the PMU location. The closed-loop behavior deteriorates as the operating condition drifts away from the nominal. Nonetheless, significantly better response is achieved under limited bandwidth availability as compared to the conventional approach of communicating the measured outputs. The deterioration is quantified in terms of the difference between the nominal and the off-nominal condition.
\end{abstract}

Index Terms-Communication, Bandwidth, Data rate, PMU, FACTS, Observer, State-feedback

\section{List of ABBREVIATIONS AND NotATIONS}

\begin{tabular}{|c|c|}
\hline PMU & Phasor Measurement Unit \\
\hline OSC & Observer Driven System Copy \\
\hline $\mathrm{CF}$ & Conventional Feedback \\
\hline FACTS & Flexible AC Transmission Systems \\
\hline TCSC & Thyristor Controlled Series Capacitor \\
\hline$G_{n}$ & $\begin{array}{l}\text { Reduced order state space model of power } \\
\text { system at nominal condition (for system copy) }\end{array}$ \\
\hline$G_{i}$ & $\begin{array}{l}\text { Reduced order state space model of power } \\
\text { system at } i^{t h} \text { off-nominal condition }\end{array}$ \\
\hline$L$ & Observer gain vector \\
\hline$K$ & State feedback gain vector \\
\hline$\sigma$ & $\begin{array}{l}\text { Time interval between consecutive samples } \\
\text { arriving at control center }\end{array}$ \\
\hline$x_{i}$ & $\begin{array}{l}\text { State vector of reduced power system model } \\
\text { under } i^{t h} \text { off-nominal condition }\end{array}$ \\
\hline $\bar{x}$ & State vector estimated by the observer \\
\hline$x_{n}$ & State vector estimated by system copy \\
\hline$t_{k}$ & Time instant of state resetting in copy \\
\hline$x_{0}$ & Observer estimated state at time $t_{k}$ \\
\hline$u(t)$ & Control input to the actuator \\
\hline
\end{tabular}

Support from the EPSRC, UK under grant EESC P11121 and Royal Academy of Engineering under Researcher Exchange Program in gratefully acknowledged.

N. Chaudhuri and B. Chaudhuri are with the Control and Power Research group, Imperial College London, London, UK (e-mail: n.chaudhuri@imperial.ac.uk, b.chaudhuri@imperial.ac.uk).

D. Chakraborty is with Indian Institute of Technology Bombay, Mumbai, India (e-mail: dc@ee.iitb.ac.in).

\author{
$x_{0}^{\prime} \quad$ Actual state of reduced power system \\ model at time $t_{k}$ \\ $\bar{u}(t) \quad$ Control input calculated at the PMUs location \\ $\tilde{A}, \tilde{B}, \tilde{C}$ Deviation in actual operating condition \\ from nominal \\ $e(t) \quad$ Error between observer and estimated \\ (by system copy) states \\ $E(t) \quad$ Error between estimated (by system copy) \\ and actual states of reduced model \\ $\|\cdot\| \quad$ Euclidian norm of a vector or a matrix \\ $t^{*} \quad$ Time instant when $\|E(t)\|$ is maximum
}

\section{INTRODUCTION}

$\mathbf{F}$ ACTS controllers could potentially be more effective with feedback signals from the distant phasor measurement units (PMUs) [1], [2], [3], [4], [5]. Conventional feedback (CF) strategy - wherein the measured PMU signals are transmitted via communication links to the control centers - is usually used. Although the existing communication infrastructure has primarily been used only for monitoring and discrete control [6], [7], [8], some utilities have shown interest in using this for closed loop continuous control. One of the major concerns, however, is the risk associated with occasional problems in the communication links.

Currently both wired (e.g. telephone lines, fibre-optic, power lines) and wireless (e.g. satellites, microwave) options are employed for the wide-area measurement systems (WAMS) [9]. Use of the telephone lines are common but provides a relatively low data rate due to the isolation requirements at the substations. Power line communication is emerging as a preferred option because it provides improved bit-errorrate [10] and offers about 4 Mbps bandwidth (BW) via the existing electric supply grid. Fibre-optic links are used by many utilities to exploit the high available BW (more than 50 Mbps). Utilities like Bonneville Power Administration (BPA) have started replacing their point to point analog microwave links with the fibre-optic communication networks [11]. Lowearth orbiting satellite technology can be an attractive option but suffers from narrow BW and associated problem of latency.

Utilities with years of experience with WAMS, are contemplating wider use of networked communication (e.g. UDP Multicast) in place of dedicated serial communication in future. The idea is to utilize the available BW partly for WAMS usage and partly for providing other data intensive services like video-conference facility [12]. Svenska Kraftnt (Swedish National Grid) has already explored the possibility of using 
a wide-area network (WAN) [13] to deliver multiple services like disturbance recording, breaker supervision, control, maintenance and wide-area protection [14], [15]. This highlights an exciting prospect in favor of the fibre-optic technology which incurs high initial investment - but provides massive BW and inherent immunity to the radio frequency. Similarly, there are plans to share the power line communication for WAMS, substation networking [16] and broadband service [17]. A recent paper on latency computation for a hypothetical wide-area control in the context of Western Electricity Coordinating Council (WECC) system conjectured a hierarchical configuration of data communication [18]. Possible use of the networked communication was indicated for implementation of such control with a large number of sensory signals from diverse geographical locations communicated to many distant zonal data concentrators [18].

With networked communication likely to be more common, impact of the data rate on the FACTS controllers is a matter of concern. It should be reiterated that limited data rates, although not common in state-of-the-art dedicated serial links, is more of a possibility in shared networked communication especially, with growing traffic through the bandwidth constrained channels. In this context an architecture for effective FACTS controllers is proposed here to deal with occasional bandwidth constrained communication.

Evidently, there is a tradeoff between the data rate and the performance of conventional feedback (CF) control. Better response and stability margins are obtained by having feedback measurements in a timely manner [19]. Dedicated WAMS infrastructure typically uses a fast data rate of 25 to 60 samples per second (samples/s) [9], [6] which is more than adequate for control of low frequency $(0.1$ to $2.0 \mathrm{~Hz})$ oscillations. However, with lower data rates there could be adverse impacts on the closed-loop behavior. A case study is presented in Section IV-A to show that the response with $\mathrm{CF}$ deteriorates quite significantly below a data rate of 10 samples/s.

The architecture suggested here is based on a predictorcorrector approach to achieve satisfactory closed-loop control under constrained data rate situation. This architecture is referred to as the observer driven system copy (OSC) approach for the rest of the paper. It is to be noted that conventional feedback (CF) should be used as usual under normal data rates. The proposed OSC architecture would be employed only below a certain threshold data rate as indicated by the timestamp information at either end [20].

Two reduced order linearized models of the power system around the nominal condition, known as system copy, are considered at the PMU and the actuator locations. The first copy is employed to create an asymptotic observer using the measured PMU output. This observer estimates the states of the reduced system which are communicated to the actuator location. The states of the second copy at the actuator location are reset by the fresh samples received from the observer. During the inter-sample interval the states are allowed to evolve on their own. The basic idea is to use the knowledge of nominal system to approximate the actual behavior during the time intervals when data is not available [19], [21], [22].

It is intuitive that the effectiveness of the OSC architecture would depend to a large extent on the difference between the actual operating condition and the one considered for the system copy. The deterioration is quantified in terms of the difference between the linearized systems at nominal and off-nominal operating conditions. Nonlinear simulation results for a range of operating scenarios are presented to verify this linear analysis. These results are compared against a conventional feedback (CF) control for different data rates. Despite the deterioration under off-nominal conditions, an OSC produces significantly better response than a CF with limited data rates.

The main contributions of this paper are:

- Investigate the impact of low data rates on FACTS controllers working with bandwidth constrained communication

- Propose an architecture for the FACTS controllers to deal with low data rates

- Analyze the impact of difference between the nominal and off-nominal conditions on the performance

- Compare the behavior of a conventional feedback (CF) and the proposed OSC architecture under bandwidth constrained communication

It should be pointed out that this paper does not propose the use of low data rate as the conventional output feedback with adequate data rate is always recommended from robustness point of view. However, under unusual circumstances leading to data rates (detected from time-stamp information at both ends [20]) below a threshold, it is preferable to switch to the proposed OSC, rather than continuing with $\mathrm{CF}$.

The rest of the paper is organized as follows. Following this introductory section, the principles of the OSC architecture is described in Section II. Quantification of the deterioration under off-nominal conditions is presented in Section III. A case study on a 16-machine, 5-area test system is presented in Section IV to illustrate the effectiveness of the OSC approach under low data rate situations.

\section{ObSERVER Driven System Copy (OSC) ApProACH}

A conventional feedback (CF) is usually employed where measured signals from the remote PMUs are communicated to the FACTS controller, see Fig. 1. Here the rate at which the data is transmitted is critical for ensuring a satisfactory closed-loop behavior. Data rates lower than a threshold could lead to unacceptable system response as illustrated in Sec IVA. The OSC approach addresses this problem by exploiting the knowledge of the nominal system to predict the actual behavior between two consecutive data samples. As illustrated in Fig. 1, the proposed OSC architecture could be switched on below a certain data rate.

A predictor-corrector approach [22], [21] is applied here to estimate (predict) the states of the reduced order linearized nominal system model (referred to as system copy) at the actuator location. These are updated (corrected) periodically at a lower rate (depending on feedback data rate) with the most recent states estimated by an observer at the PMU location as shown in Fig. 1. 


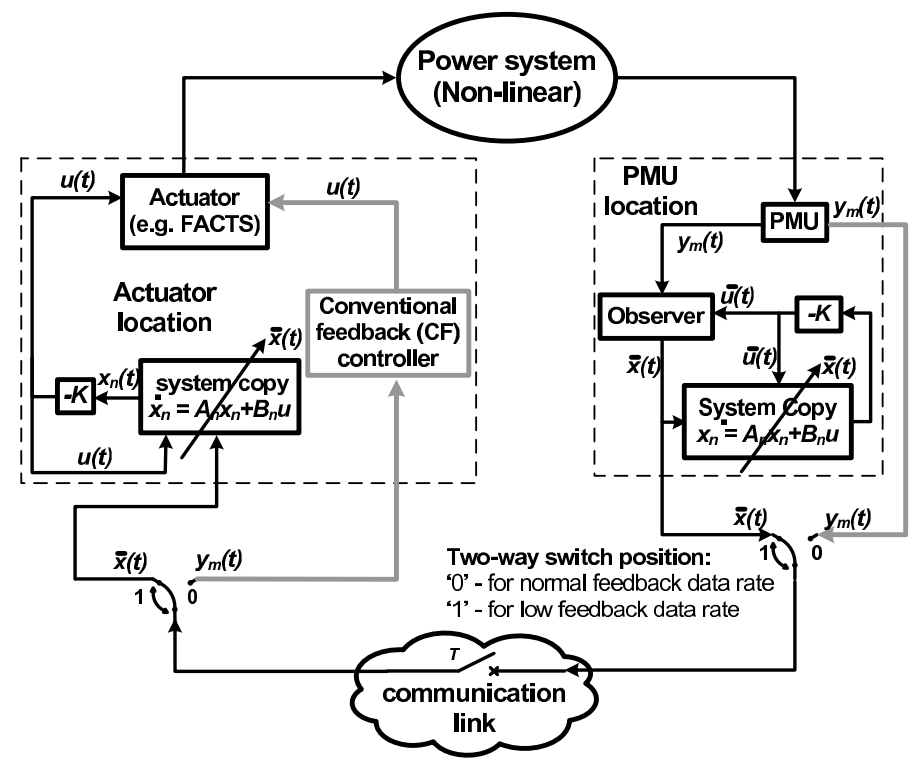

Fig. 1. Overall architecture including conventional feedback (CF) (in grey) and observer driven system copy (OSC) approach

The reduced order linearized model of the power system around the nominal operating condition $G_{n}$ is given by:

$$
G_{n}=\left[\begin{array}{c|c}
A_{n} & B_{n} \\
\hline C_{n} & 0
\end{array}\right]
$$

where, $A_{n} \in \Re^{m \times m}, B_{n} \in \Re^{m \times n}$ and $C_{n} \in \Re^{p \times m}$. Reduced order linearized model under $i^{\text {th }}$ operating condition $G_{i}$ (e.g. corresponding to a line outage) is denoted as:

$$
G_{i}=\left[\begin{array}{c|c}
A_{i} & B_{i} \\
\hline C_{i} & 0
\end{array}\right]
$$

where, $A_{i}=A_{n}+\tilde{A}, B_{i}=B_{n}+\tilde{B}, C_{i}=C_{n}+\tilde{C}$ and $\tilde{A}$, $\tilde{B}, \tilde{C}$ represent the deviation around the nominal operating condition. The states of $G_{n}$ and $G_{i}$ are denoted as $x_{n}(t)$ and $x_{i}(t)$, respectively. Exogenous disturbances could also be incorporated in (2), if required.

An observer (3) at the PMU location estimates the states $\bar{x}$ of the reduced order system which are transmitted through the communication network to the controller. The state equation of the observer is:

$$
\dot{\bar{x}}=\left(A_{n}-L C_{n}\right) \bar{x}+B_{n} \bar{u}+L C_{i} x_{i}
$$

Note that the observer at the PMU location requires knowledge of the control input $u(t)$ which is calculated $(\bar{u}(t))$ using the system copy model and the state-feedback gain vector $K$, see Fig. 1. The system copy at the actuator location is based on the nominal model described by the following equation:

$$
\dot{x}_{n}(t)=A_{n} x_{n}(t)+B_{n} u(t)
$$

Depending on the data rate available, the communication channel transmits data between the remote observer location and the local system copy based controller, only at time instants $\left\{t_{k}\right\}_{k=0}^{\infty}$. It is assumed that this "sampling" of the remote data occurs at equally spaced intervals so that the intersample time is $t_{k+1}-t_{k}=\sigma \forall k=0,1, \ldots$. Hence the states of (4) are reset to the states estimated by (3) at the sampling instants $\left\{t_{k}\right\}_{k=0}^{\infty}$.

$$
x_{n}\left(t_{k}\right)=\bar{x}\left(t_{k}\right) \text { for all } k=0,1,2, \ldots
$$

The input used to control $G_{i}$ is synthesized using the nominal model (4) and (5) according to the following equation:

$$
u(t)=-K x_{n}(t)
$$

where $K \in \Re^{1 \times m}$ is the state feedback gain vector designed based on the nominal system model $G_{n}$.

During time interval $\sigma$, when the reduced order system states are not available from the PMU location (i.e. $\mathrm{T}$ is open, see Fig. 1) the system copy predicts the states. Upon arrival of the next available sample of $\bar{x}(t)$ the states of the system copy are corrected/reset leading to a switched control strategy. This setup is referred to as observer driven system copy (OSC) in this paper.

Combining equations (2), (4), (6) and (3) the overall system dynamics during the time interval $t \in\left[t_{k}, t_{k+1}\right), t_{k+1}-t_{k}=\sigma$ can be described as:

$$
\left[\begin{array}{c}
\dot{x}_{i} \\
\dot{x}_{n} \\
\dot{\bar{x}}
\end{array}\right]=\left[\begin{array}{ccc}
A_{i} & -B_{i} K & 0 \\
0 & A_{n}-B_{n} K & 0 \\
L C_{i} & -B_{n} K & A_{n}-L C_{n}
\end{array}\right]\left[\begin{array}{c}
x_{i} \\
x_{n} \\
\bar{x}
\end{array}\right]
$$

with the additional condition imposed by (5) at all $t_{k}$. The initial condition $x_{i}(0)$ is usually unknown while the initial conditions for the nominal and the observer states are assumed to be zero $x_{n}(0)=0$ and $\bar{x}(0)=0$.

Following [22], the error $e=\bar{x}-x_{n}$ is defined as the difference between the nominal and estimated (observer) states. Using a linear transformation (7) can be re-written in terms of the error $e(t)$ as follows:

$$
\left[\begin{array}{c}
\dot{x}_{i} \\
\dot{\bar{x}} \\
\dot{e}
\end{array}\right]=\left[\begin{array}{ccc}
A_{i} & -B_{i} K & B_{i} K \\
L C_{i} & A_{n}-L C_{n}-B_{n} K & B_{n} K \\
L C_{i} & -L C_{n} & A_{n}
\end{array}\right]\left[\begin{array}{c}
x_{i} \\
\bar{x} \\
e
\end{array}\right]
$$

It can be proved that the system (8) is globally, exponentially stable around the solution $\left[\begin{array}{lll}x_{i} & \bar{x} & e\end{array}\right]^{T}=\left[\begin{array}{lll}0 & 0 & 0\end{array}\right]^{T}$ if and only if the eigenvalues of (9) lie inside the unit circle [22].

$$
\Lambda=\left[\begin{array}{lll}
I & 0 & 0 \\
0 & I & 0 \\
0 & 0 & 0
\end{array}\right] e^{\Gamma \sigma}\left[\begin{array}{lll}
I & 0 & 0 \\
0 & I & 0 \\
0 & 0 & 0
\end{array}\right]
$$

where $\Gamma$ is the overall state matrix in (8). Maximum allowable update interval $\sigma$ i.e. minimum data rate can be obtained from the stability of (8).

It should be noted that while (9) characterizes global exponential stability of the linear switched system (8), it does not formally establish the stability of the non-linear power system under switching [23]. However, for practical purposes, stability and performance is guaranteed through extensive simulations reported in Section IV.

\section{INTER-SAMPLE ERROR ESTIMATE}

It was shown in the previous section that the asymptotic behavior of the OSC approach is guaranteed to be exponentially stable under appropriate conditions. However, it would 
be useful to estimate the deterioration under off-nominal conditions, which depends on the evolution of the states of $G_{i}$ during the period between two consecutive feedback samples. This is quantified in this section in terms of the difference between the nominal and off-nominal operating conditions.

From dynamics of the combined nominal, off-nominal and observer systems (7) during the inter-sample period $\left[t_{k}, t_{k+1}\right)$, it is observed that the responses of $x_{i}(t)$ and $x_{n}(t)$ are uncoupled with that of the observer $\bar{x}(t)$. Hence, the left upper block can be considered separately for analysis during $t \in\left[t_{k}, t_{k+1}\right)$. Thus, neglecting the observer dynamics without loss of generality, (7) can be rewritten as:

$$
\left[\begin{array}{c}
\dot{x}_{i}(t) \\
\dot{x}_{n}(t)
\end{array}\right]=\left[\begin{array}{cc}
A_{i} & -B_{i} K \\
0 & A_{n}-B_{n} K
\end{array}\right]\left[\begin{array}{c}
x_{i}(t) \\
x_{n}(t)
\end{array}\right]
$$

The initial conditions are the states at the previous available sampling instant $t_{k}$. Assuming

$$
\left[\begin{array}{l}
x_{i}\left(t_{k}\right) \\
x_{n}\left(t_{k}\right)
\end{array}\right]=\left[\begin{array}{l}
x_{0}^{\prime} \\
x_{0}
\end{array}\right]
$$

It is to be noted that the state $x_{n}\left(t_{k}\right)$ is reset to the estimated observer state $\bar{x}\left(t_{k}\right)$ according to (5). Solution of (10) gives:

$$
\left[\begin{array}{c}
x_{i}(t) \\
x_{n}(t)
\end{array}\right]=e^{\Delta\left(t-t_{k}\right)}\left[\begin{array}{l}
x_{0}^{\prime} \\
x_{0}
\end{array}\right], t \in\left[t_{k}, t_{k+1}\right)
$$

where,

$$
\Delta=\left[\begin{array}{cc}
A_{i} & -B_{i} K \\
0 & A_{n}-B_{n} K
\end{array}\right]
$$

Equation (11) represents the temporal evolution of system states of the reduced order model and those predicted by the system copy. The state trajectory of system copy with initial state $x_{0}$ can be expressed as:

$$
x_{n}(t)=e^{\left(A_{n}-B_{n} K\right)\left(t-t_{k}\right)} x_{0} \text { for } t \in\left[t_{k}, t_{k+1}\right)
$$

An analytical expression for the trajectory of the states of the reduced order linearized power system model is derived as follows. Transforming (11) to Laplace domain we get:

$$
\left[\begin{array}{l}
X_{i}(s) \\
X_{n}(s)
\end{array}\right]=\mathcal{L}\left\{e^{\Delta\left(t-t_{k}\right)}\left[\begin{array}{l}
x_{0}^{\prime} \\
x_{0}
\end{array}\right]\right\}=\Xi\left[\begin{array}{l}
x_{0}^{\prime} \\
x_{0}
\end{array}\right]
$$

where,

$\Xi=\left[\begin{array}{cc}\left(s I-A_{i}\right)^{-1} & -\left(s I-A_{i}\right)^{-1} B_{i} K\left(s I-A_{n}+B_{n} K\right)^{-1} \\ 0 & \left(s I-\left(A_{n}-B_{n} K\right)\right)^{-1}\end{array}\right]$

Hence, $X_{i}(s)$ can be written as:

$$
\begin{aligned}
& X_{i}(s)=\left(s I-A_{i}\right)^{-1} x_{0}^{\prime} \\
& -\left(s I-A_{i}\right)^{-1} B_{i} K\left(s I-A_{n}+B_{n} K\right)^{-1} x_{0}
\end{aligned}
$$

which simplifies to:

$$
\begin{aligned}
& X_{i}(s)=\left(s I-A_{i}\right)^{-1}\left(x_{0}^{\prime}-x_{0}\right) \\
& +\left(s I-A_{i}\right)^{-1}\left[I-B_{i} K\left(s I-A_{n}+B_{n} K\right)^{-1}\right] x_{0}
\end{aligned}
$$

Equation (17) can be further simplified to:

$$
\begin{aligned}
& X_{i}(s)=\left(s I-A_{n}+B_{n} K\right)^{-1} x_{0}+\left(s I-A_{i}\right)^{-1}\left(x_{0}^{\prime}-x_{0}\right) \\
& +\left(s I-A_{i}\right)^{-1}(\tilde{A}-\tilde{B} K)\left(s I-A_{n}+B_{n} K\right)^{-1} x_{0}
\end{aligned}
$$

Notably $\tilde{A}$ and $\tilde{B}$ represent the deviation of the dynamic model embedded in system copy from the linearized system model (corresponding to a particular operating condition). Thus the actual system states are given by:

$$
\begin{aligned}
& x_{i}(t)=e^{\left(A_{n}-B_{n} K\right)\left(t-t_{k}\right)} x_{0}+e^{A_{i}\left(t-t_{k}\right)}\left(x_{0}^{\prime}-x_{0}\right) \\
& +\int_{t_{k}}^{t} e^{A_{i}(t-\tau)}(\tilde{A}-\tilde{B} K) e^{\left(A_{n}-B_{n} K\right) \tau} x_{0} d \tau
\end{aligned}
$$

The error between the reduced order linearized system state trajectory and that estimated by system copy can be expressed as:

$$
\begin{aligned}
& E(t):=x_{i}(t)-x_{n}(t) \\
& =e^{A_{i}\left(t-t_{k}\right)}\left(x_{0}^{\prime}-x_{0}\right)+\int_{t_{k}}^{t} e^{A_{i}(t-\tau)}(\tilde{A}-\tilde{B} K) e^{\left(A_{n}-B_{n} K\right) \tau} x_{0} d \tau
\end{aligned}
$$

It can be observed that the error term consists of two components. The first term represents the deviation of linearized system state from its asymptotic estimate computed by the observer at $t=t_{k}$. The second term arises due to the difference between actual power system operating condition and the system copy model. As expected, if both the initial condition error and the model mismatch can be reduced to zero, i.e.

$$
x_{0}^{\prime}=x_{0} ; \quad \tilde{A}=0, \tilde{B}=0
$$

the error $E(t)$ ceases to exist. However, because of the changes in the operating conditions in practical systems, (21) does not hold good and there is always a finite error.

Assuming a stable open-loop system there are constants $k_{1}>0$ and $\alpha_{1}>0$ such that for any vector $c_{1} \in \Re^{m}$ :

$$
\left\|e^{A_{i} t} c_{1}\right\| \leq k_{1} e^{-\alpha_{1} t}\left\|c_{1}\right\|
$$

Moreover, the closed-loop nominal system is stable and welldamped with the designed controller implying there exists constants $k_{2}>0$ and $\alpha_{2}>0$ such that for any vector $c_{2} \in \Re^{m}$ :

$$
\left\|e^{\left(A_{n}-B_{n} K\right) t} c_{2}\right\| \leq k_{2} e^{-\alpha_{2} t}\left\|c_{2}\right\|
$$

Using (22) and (23) an estimate of the error $E(t)$ can be derived as follows:

$$
\begin{aligned}
& \|E(t)\| \leq\left\|e^{A_{i}\left(t-t_{k}\right)}\right\|\left\|\left(x_{0}^{\prime}-x_{0}\right)\right\| \\
& +\left\|\int_{t_{k}}^{t} e^{A_{i}(t-\tau)}(\tilde{A}-\tilde{B} K) e^{\left(A_{n}-B_{n} K\right) \tau} x_{0} d \tau\right\| \\
& \Rightarrow\|E(t)\| \leq k_{1}\left\|\left(x_{0}^{\prime}-x_{0}\right)\right\| e^{-\alpha_{1}\left(t-t_{k}\right)} \| \\
& +k_{1} k_{2}\|(\tilde{A}-\tilde{B} K)\|\left\|x_{0}\right\| \int_{t_{k}}^{t} e^{-\alpha_{1}(t-\tau)} e^{-\alpha_{2} \tau} d \tau \\
& =k_{1}\left\|\left(x_{0}^{\prime}-x_{0}\right)\right\| e^{-\alpha_{1}\left(t-t_{k}\right)} \\
& +k_{1} k_{2}\|(\tilde{A}-\tilde{B} K)\|\left\|x_{0}\right\| \frac{\left[e^{-\alpha_{2}\left(t-t_{k}\right)}-e^{-\alpha_{1}\left(t-t_{k}\right)}\right]}{\left(\alpha_{1}-\alpha_{2}\right) e^{\alpha_{2} t_{k}}}
\end{aligned}
$$

It is to be noted that the consecutive asymptotic estimate of the reduced order linearized system states reset the system copy over finite intervals of time. Assuming that the eigenvalues of (9) lie inside the unit circle, the system (8) is globally, exponentially stable. Hence it follows that the sampling instant error is $\left\|x_{i}\left(t_{k}\right)-x_{n}\left(t_{k}\right)\right\| \rightarrow 0$ as $k \rightarrow \infty$. However, the maximum value of the error during the inter-sample interval, $t \in\left[t_{k}, t_{k+1}\right)$ between two consecutive samples is of interest. Suppose the error attain the peak value at some $t_{k}^{*} \in\left[t_{k}, t_{k+1}\right]$. 


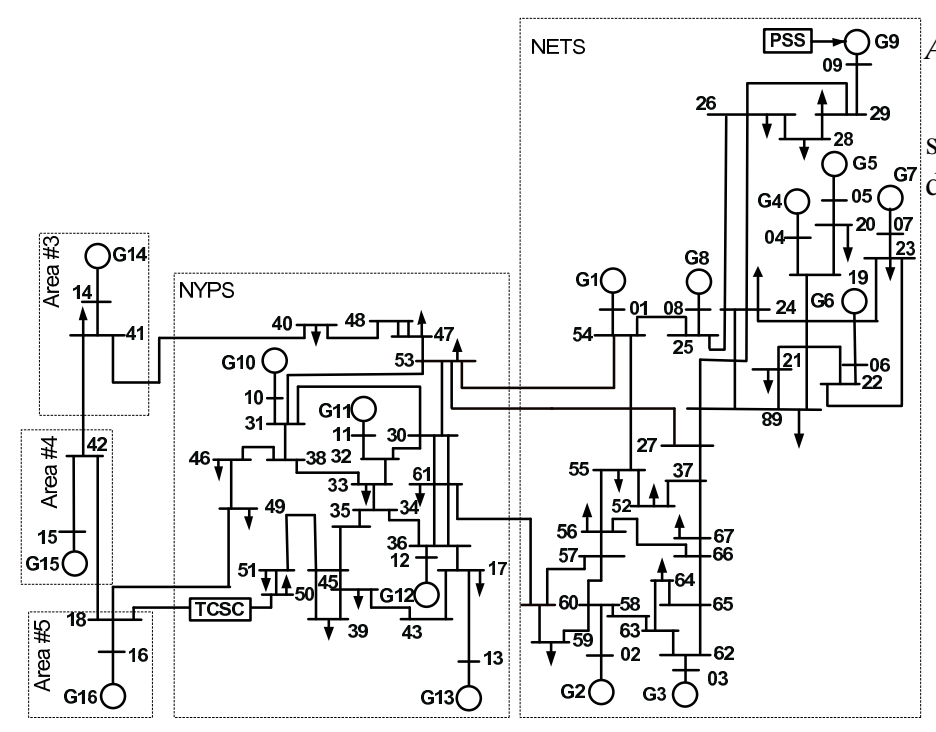

Fig. 2. Test system : 16-machine, 5-area system with a TCSC

Assuming $\left(x_{0}^{\prime}-x_{0}\right)=0$, from (24), it can be seen that the maximum error norm is proportional to the model mismatch:

$$
\left\|E\left(t^{*}\right)\right\| \propto\|\tilde{A}-\tilde{B} K\|
$$

\section{CASE STUdY}

To illustrate the effectiveness of the proposed OSC approach under limited data rate conditions, a case study was carried out on a 16-machine, 5-area test system, shown in Fig. 2. A detailed description of the study system including the machine, excitation systems and the network parameters can be found in [24]. A thyristor controlled series capacitor (TCSC) is installed on the tie-line connecting the buses 18 and 50 and is used to damp power oscillations with the real power flow in the line 45-35 as the feedback signal [25].

For a conventional feedback (CF) controller, the measured signals from PMUs $\left(y_{m}\right)$ are communicated to the controller at the actuator location. Here such a controller is designed using linear quadratic regulator (LQR) approach [26] based on a 10th order reduced model of the nominal power system.

For the proposed OSC, the states of the reduced order system (not the measured outputs) estimated by the observer are communicated over the network. A reduced order system model (system copy) is used to calculate the control input $(\bar{u}(t))$ required by the observer at the PMU location, see Fig. 1 . A system copy containing the same reduced order model drives a state feedback controller at the actuator location as described in Section II. In this exercise, a balanced truncation approach [27] is used to obtain the reduced order nominal model of the power system. For large scale power systems, subspace based techniques for model reduction could be employed.

A low pass filter is used at the output of the system copy to suppress sharp changes in the control signal due to periodic reset with most recent states. A 20 ms latency is considered in the communication channel.

\section{A. Impact of low data rate on $C F$}

Impact of low data rates on the $\mathrm{CF}$ is illustrated in this subsection. The closed-loop response was tested for different data rates in the range 1 sample/s $(1 / \mathrm{s})$.
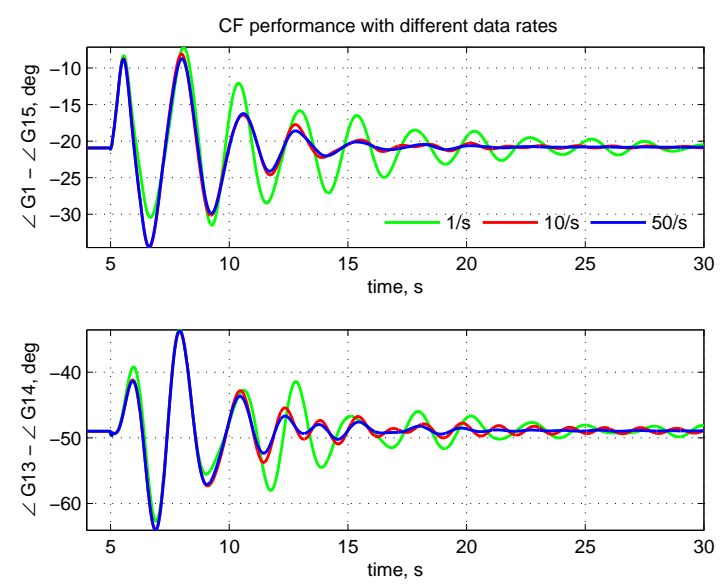

Fig. 3. Comparison of $\mathrm{CF}$ for different data rates with a self-clearing fault for $80 \mathrm{~ms}$ near bus 60

System responses with the $\mathrm{CF}$ are compared for data rates of 50, 10 and 1 samples/s following a self-clearing fault for 80 ms near bus 60 , see Fig. 3. With 10 samples/s the behavior is very close to that $50 \mathrm{samples} / \mathrm{s}$ while it is much worse with 1 sample/s. Here self clearing fault is considered deliberately to rule out any possible deterioration due to change in operating condition.
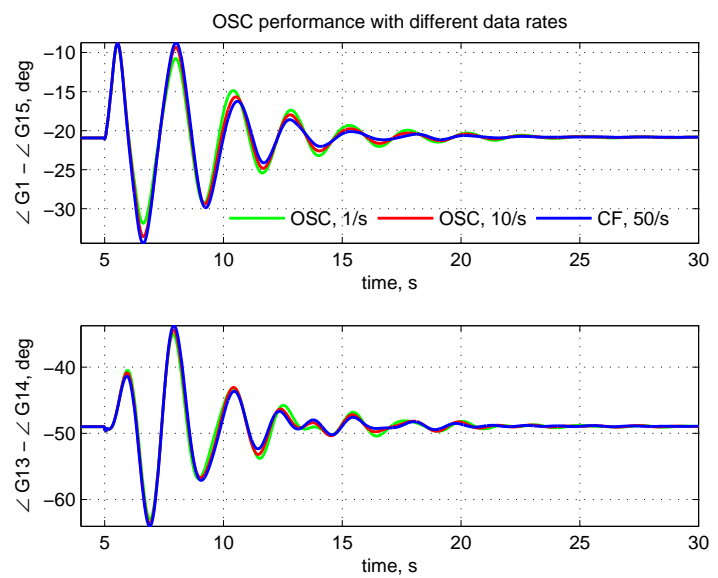

Fig. 4. Comparison of $\mathrm{CF}$ at nominal data rate (50 samples/s) vs OSC with lower data rate for a self-clearing fault for $80 \mathrm{~ms}$ near bus 60

\section{B. OSC with low data rate}

The above case study was repeated with the proposed OSC approach. Fig. 4 compares the OSC with low data rates against the CF using a typical PMU rate of 50 samples/s. For a selfclearing fault for $80 \mathrm{~ms}$ near bus 60 , the OSC produces almost identical response as the CF. This implies that the system copy could approximate the actual behavior quite closely during intervals of absence of the feedback data. 
It is to be noted that a system copy having the same order as the linearized plant would exactly replicate the behavior in open-loop condition. However, some deviations are expected in the simulation results due to non-linearities and model reduction effects. Drift in the operating condition away from the nominal will result in growing difference between the estimated response by the observer and the system copy leading to deterioration of the OSC performance shown in the next subsection.

\section{Effect of operating condition on OSC}

Performance of the OSC under three different line outage scenarios were compared against the nominal condition. Clearly, there are two factors that are expected to cause a poorer behavior:

- difference between the system copy model (nominal) and the actual operating condition

- controller is design for the nominal condition

Although the impact of these factors can not be decoupled completely, the effect of the deviation of the system operating condition on the OSC can be captured by:

- comparing the OSC against CF at nominal data rate (2560 samples/s) as a benchmark (to take care of the effect of controller)

- illustrating OSC performance with data rates as low as 1 sample every $2 \mathrm{~s}$ (this will rely predominantly on the proximity of the system copy model and the actual operating condition)
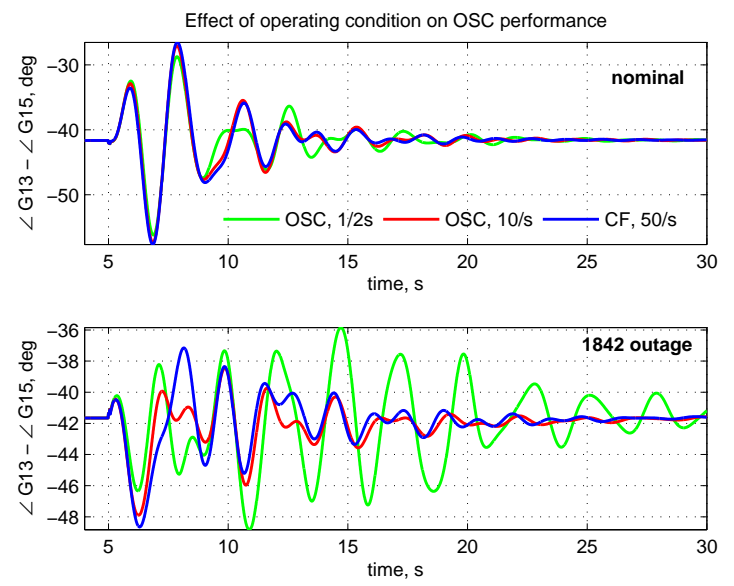

Fig. 5. Effect of change in operating condition on OSC performance

The system behavior with the $\mathrm{CF}$ for different operating conditions are illustrated in Figs. 5 and 6 which reveal the following:

- at nominal condition, OSC with 1 sample every $2 \mathrm{~s}$ $(1 / 2 \mathrm{~s})$ behaves marginally differently than $\mathrm{CF}$ with 50 samples/s (see Fig. 5) due to system nonlinearities and model reduction effects

- CF with 50 samples/s produces satisfactory behavior under different operating conditions indicating a reasonable robustness of the controller (see Figs. 5, 6)
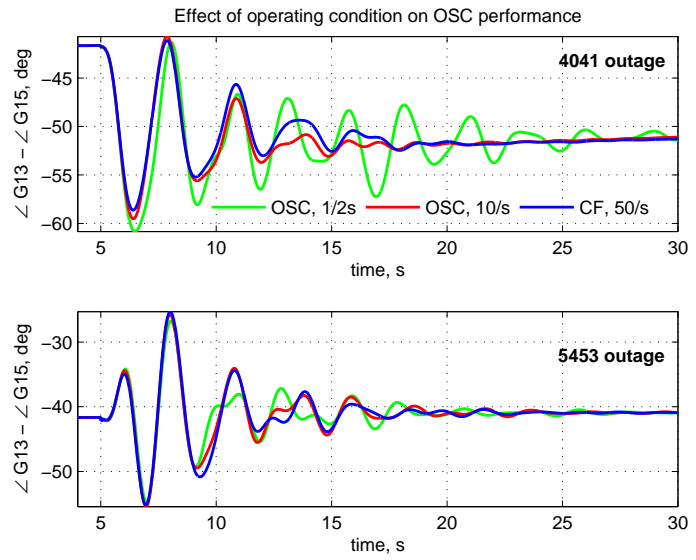

Fig. 6. Effect of change in operating condition on OSC performance

- compared to the CF, considered as benchmark, the performance with OSC becomes poorer with lower data rates due to increasing reliance on accuracy of system copy

- performance with OSC is worst for line 18-42 outage (see Fig. 5) followed by line 40-41 and line 54-53 outages (see Fig. 6)

It is not straightforward to justify the trends in simulation with the analysis in Section III primarily, due to the effect of non-linearities. However, the above observations qualitatively agree with the measure of the error bound in (25). The calculated values of the error bounds based on the linearized models for different outage conditions are given in Table I. It is important to note that, the objective of this exercise was to illustrate the working principle of the proposed OSC approach in a nonlinear environment and validate whether it behaves as expected from the linear control theory.

TABLE I

MEASURE OF MAXIMUM ERROR BOUND

\begin{tabular}{|c||c|}
\hline Outage of line & $\|\tilde{A}-\tilde{B} K\|$ \\
\hline \hline $18-42$ & 176 \\
\hline $40-41$ & 235 \\
\hline $54-53$ & 97 \\
\hline $60-61$ & 243 \\
\hline
\end{tabular}

\section{OSC vs CF at low data rate}

This subsection shows that the performance with the OSC, even under off-nominal operating scenarios, is consistently better compared to the $\mathrm{CF}$ if low data rates are used. Since the behavior of the CF is poor with 1 sample/s at the nominal condition (see Sec IV-A) and more so for the outage conditions - a minimum data rate of 2 samples/s was used.

Fig. 7 compares the system responses with CF and OSC for a three phase fault for $80 \mathrm{~ms}$ near bus 60 followed by line 60-61 outage. The effectiveness of the OSC is evident in sharp contrast to the adverse impact of the low feedback rates on the CF. A closer look at the variation of the percentage compensation of the TCSC (see Fig. 7, lower subplot) reveals that the control effort with the OSC is delayed by about $0.5 \mathrm{~s}$ 

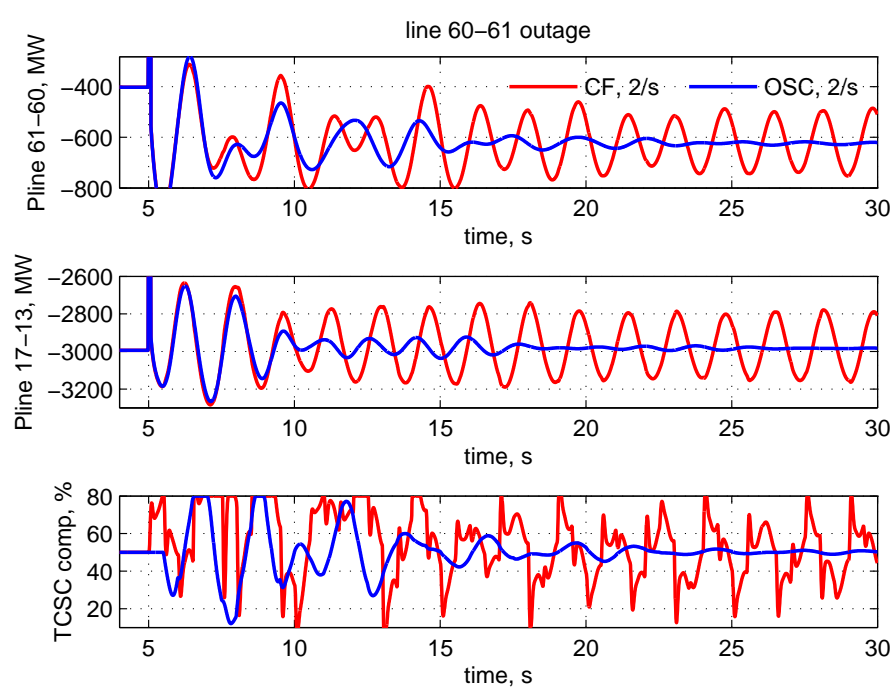

Fig. 7. Comparison between OSC and $\mathrm{CF}$ at 2 samples/s data rate for line 60-61 outage

(precisely $0.52 \mathrm{~s}$ ) due to the delayed arrival of the first sample of non-zero states from the PMU location to the control center. Figs. 8 and 9 show the robustness of the proposed technique
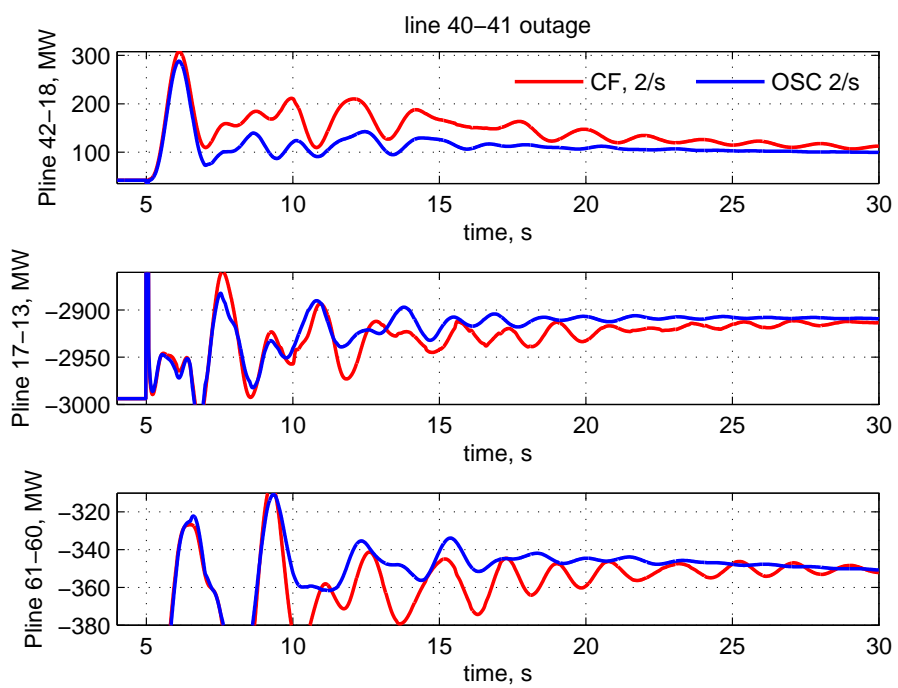

Fig. 8. Comparison between OSC and CF at 2 samples/s data rate for line 40-41 outage

across the different operating conditions (line 40-41 and 1842 outage). The case studies demonstrate that the proposed OSC approach produces a satisfactory closed-loop behavior under different operating scenarios. On the other hand the $\mathrm{CF}$ results in unacceptable response with low data rates. Similar observation holds good for line 54-53 outage illustrated in Fig. 10

\section{E. OSC at very low data rate}

With data rates typically in the range of 1-2 samples per second (1-2/s) [6], the OSC approach resulted in a satisfactory closed-loop system response even under the off-nominal operating scenarios. Fig. 11 compares the performance of the CF
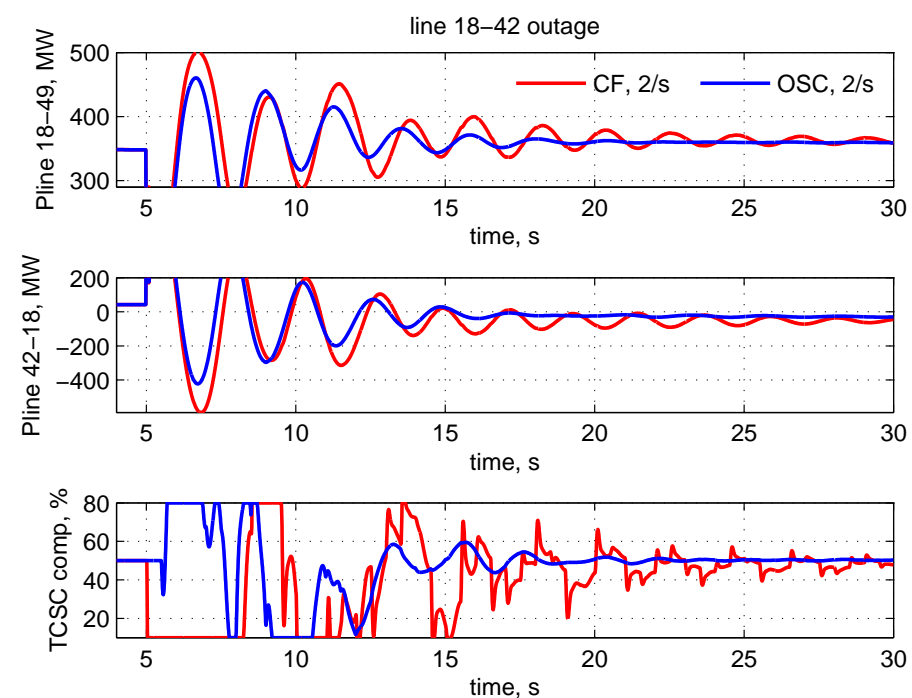

Fig. 9. Comparison between OSC and CF at 2 samples/s data rate for line 1842 outage
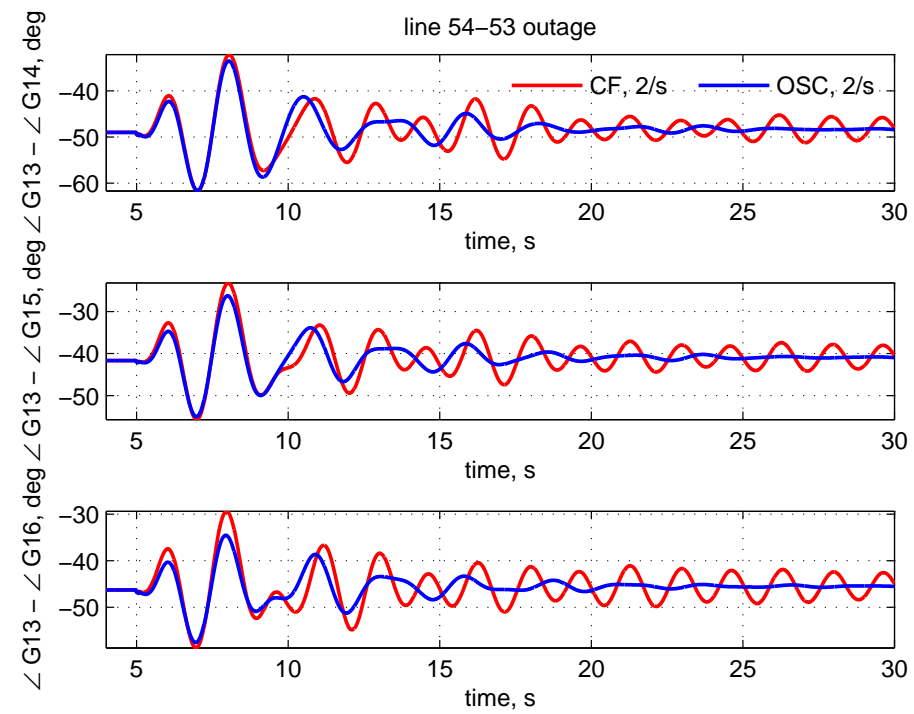

Fig. 10. Comparison between OSC and CF at 2 samples/s data rate for line 54-53 outage

using a typical PMU data rate (50/s) against the OSC using a much lower rate (1/s). The system responses are comparable demonstrating the effectiveness of Othe SC approach under low feedback data rate situations.

It is to be noted that with still lower data rates (e.g. 1 sample $/ 2$ s) the OSC produces poorer responses compared to CF with 50 samples/s which is expected beyond a certain point.

\section{CONClusion}

Data feedback rate below a certain threshold is shown to have adverse effect on the FACTS controllers relying on signals from the phasor measurement units (PMUs). An observer driven system copy (OSC) architecture is suggested here to deal with low data rates of the order of 1-2 samples per seconds $(1-2 / s)$. The basic idea is to use the knowledge 

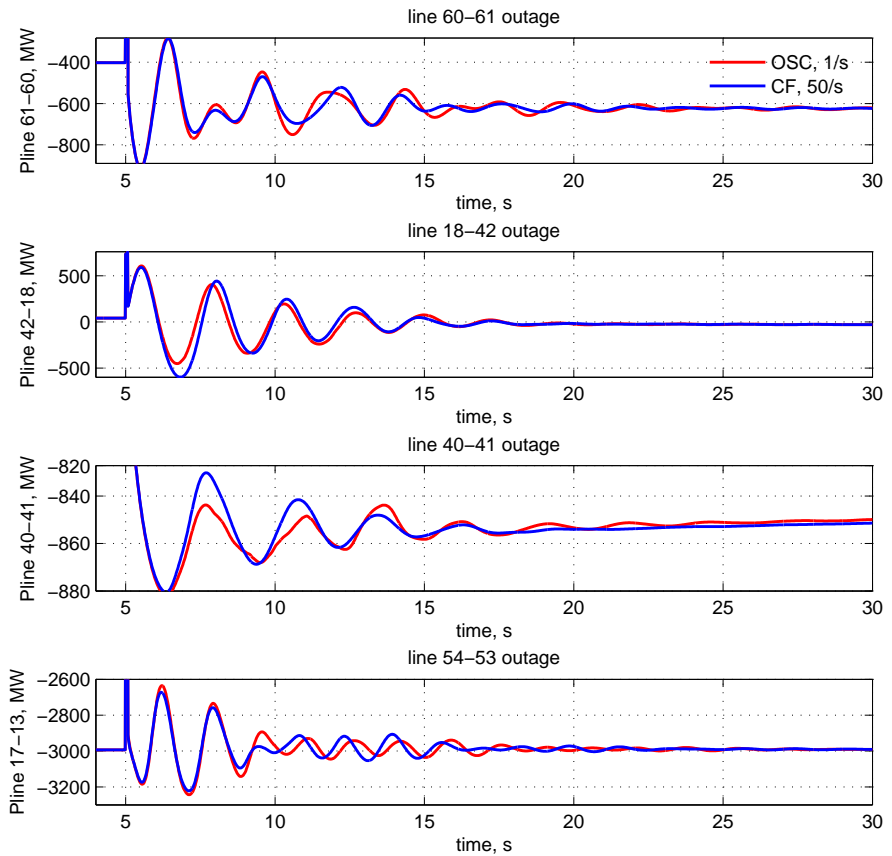

Fig. 11. Comparison of OSC at 1 sample/s data rate with $\mathrm{CF}$ at nominal (50 samples/s) data rate across different operating conditions

of the nominal system to approximate the actual behavior during intervals when data from PMUs is not available. Each time new data samples arrive, this estimate is updated by the most recent states provided by the observer at the PMU location. Of course the closed-loop behavior deteriorates as the operating condition drifts away from the nominal dynamics. Nonetheless, significantly better response compared to the conventional feedback (CF) is obtained under bandwidth constrained communication. The deterioration is quantified in terms of the difference between the nominal and the offnominal conditions.

It should be pointed out that the aim of this paper is not to propose use of low data rate as the conventional output feedback with an adequate data rate is always recommended from the robustness point of view. However, under unavoidable circumstances leading to data rates (detected from time-stamp information at both ends) below a threshold, it is preferable to switch to the proposed OSC architecture, rather than continuing with the $\mathrm{CF}$.

\section{REFERENCES}

[1] I. Kamwa, A. Heniche, G. Trudel, M. Dobrescu, R. Grondin, and D. Lefebvre, "Assessing the technical value of FACTS-based wide-area damping control loops," in proceedings of IEEE Power Engineering Society General Meeting, 2005, 2005, pp. 1734-1743 Vol. 2.

[2] J. Chow, J. Sanchez-Gasca, H. Ren, and S. Wang, "Power system damping controller design-using multiple input signals," IEEE Control Systems Magazine, vol. 20, no. 4, pp. 82-90, 2000.

[3] B. Chaudhuri and B. Pal, "Robust damping of multiple swing modes employing global stabilizing signals with a TCSC," in IEEE Power Engineering Society General Meeting, vol. 2, 2004, p. 1709.

[4] M. Aboul-Ela, A. Sallam, J. McCalley, and A. Fouad, "Damping controller design for power system oscillations using global signals," IEEE Transactions on Power Systems, vol. 11, no. 2, pp. 767-773, 1996.
[5] S. Ray, G. K. Venayagamoorthy, B. Chaudhuri, and R. Majumder, "Comparison of adaptive critic-based and classical wide-area controllers for power systems," IEEE Transactions on Systems, Man, and Cybernetics, Part B, vol. 38, no. 4, pp. 1002-1007, 2008.

[6] J. Y. Cai, H. Zhenyu, J. Hauer, and K. Martin, "Current status and experience of WAMS implementation in north america," in IEEE/PES Transmission and Distribution Conference and Exhibition: Asia and Pacific, 2005, 2005, pp. 1-7.

[7] R. E. Wilson and C. W. Taylor, "Using dynamic simulations to design the wide-area stability and voltage control system (wacs)," in IEEE PES Power Systems Conference and Exposition, 2004, pp. 100-107 vol.1.

[8] C. W. Taylor, D. C. Erickson, K. E. Martin, R. E. Wilson, and V. Venkatasubramanian, "Wacs-wide-area stability and voltage control system: R\&D and online demonstration," Proceedings of the IEEE, vol. 93, no. 5, pp. 892-906, 2005.

[9] B. Naduvathuparambil, M. C. Valenti, and A. Feliachi, "Communication delays in wide area measurement systems," in Proceedings of the ThirtyFourth Southeastern Symposium on System Theory, 2002, pp. 118-122.

[10] K. M. Dostert, "Frequency-hopping spread-spectrum modulation for digital communications over electrical power lines," IEEE Journal on Selected Areas in Communications, vol. 8, no. 4, pp. 700-710, 1990.

[11] C. W. Taylor, M. V. Venkatasubramanian, and Y. Chen, "Wide-area stability and voltage control," in VII Symposium of Specialists in Electric Operational and Expansion Planning, 2000, pp. 1-9.

[12] L. Lamarre, "At home with communications," EPRI Journal, pp. 7-15, January/February 1997.

[13] G. N. Ericsson, "Communication requirements - basis for investment in a utility wide-area network," IEEE Transactions on Power Delivery, vol. 19, no. 1, pp. 92-95, 2004.

[14] M. G. Adamiak, A. P. Apostolov, M. M. Begovic, C. F. Henville, K. E. Martin, G. L. Michel, A. G. Phadke, and J. S. Thorp, "Wide area protection: Technology and infrastructures," IEEE Transactions on Power Delivery, vol. 21, no. 2, pp. 601-609, 2006.

[15] "Analog/digital microwave considerations for electric/gas utilities," IEEE Transactions on Power Delivery, vol. 8, no. 3, pp. 798-815, 1993.

[16] D. Radford, "Spread spectrum data leap through ac power wiring," IEEE Spectrum, vol. 33, no. 11, pp. 48-53, 1996.

[17] D. Schneider, "Is this the moment for broadband over power lines?" IEEE Spectrum, vol. 46, no. 7, pp. 17-17, 2009.

[18] J. W. Stahlhut, T. J. Browne, G. T. Heydt, and V. Vittal, "Latency viewed as a stochastic process and its impact on wide area power system control signals," IEEE Transactions on Power Systems, vol. 23, no. 1, pp. 84-91, 2008.

[19] L. A. Montestruque and P. Antsaklis, "Stability of model-based networked control systems with time-varying transmission times," IEEE Transactions on Automatic Control, vol. 49, no. 9, pp. 1562-1572, 2004.

[20] N. R. Chaudhuri, S. Ray, R. Majumder, and B. Chaudhuri, "A new approach to continuous latency compensation with adaptive phasor power oscillation damping controller (pod)," IEEE Transactions on Power Systems, vol. 25, no. 2, pp. 939-946, 2010.

[21] P. V. Zhivoglyadov and R. H. Middleton, "Networked control design for linear systems," Automatica, vol. 39, no. 4, pp. 743-750, 2003.

[22] L. A. Montestruque and P. J. Antsaklis, "On the model-based control of networked systems," Automatica, vol. 39, no. 10, pp. 1837-1843, 2003.

[23] M. S. Branicky, "Stability of switched and hybrid systems," in Proceedings of the 33rd IEEE Conference on Decision and Control, 1994, vol. 4, 1994, pp. 3498-3503 vol.4.

[24] B. Pal and B. Chaudhuri, Robust control in power systems, ser. Power electronics and power systems. New York: Springer, 2005.

[25] S. Ray, B. Chaudhuri, and R. Majumder, "Appropriate signal selection for damping multi-modal oscillations using low order controllers," in proceedings of IEEE Power Engineering Society General Meeting, 2008, Pittsburgh, 2008.

[26] A. Zolotas, B. Chaudhuri, I. Jaimoukha, and P. Korba, "A study on LQG/LTR control for damping inter-area oscillations in power systems," IEEE Transactions on Control Systems Technology, vol. 15, no. 1, pp. 151-160, 2007.

[27] S. Skogestad and I. Postlethwaite, Multivariable feedback control: analysis and design. Chichester: Wiley, 1996. 